\title{
Variable Selection for Partially Linear Additive Model Based on Modal Regression Under High Dimensional Data
}

\author{
Yafeng Xia*, Lirong Zhang \\ School of Sciences, Lanzhou University of Technology, Lanzhou, P. R. China
}

\section{Email address:}

gsxyf01@163.com (Lirong Zhang)

${ }^{*}$ Corresponding author

\section{To cite this article:}

Yafeng Xia, Lirong Zhang. Variable Selection for Partially Linear Additive Model based on Modal Regression under High Dimensional Data. International Journal of Statistical Distributions and Applications. Vol. 6, No. 1, 2020, pp. 1-9. doi: 10.11648/j.ijsd.20200601.11

Received: December 19, 2019; Accepted: January 9, 2020; Published: April 17, 2020

\begin{abstract}
In this article, we focus on the variable selection for partially linear additive model under high dimensional data.Variable selection is proposed based on modal regression estimation with Adoptive Bridge Method.Using the B-spline basic function to approximate the additive function, a penalty estimation objective equation is constructed.It establishes and proves that the variable selection methods have oracle property.Numerical simulations tested the performance of the proposed methods in a finite sample and verified the significance of the proposed estimation and the variable selection methods. At the end of the article, we attach the detailed derivation of the theoretical results. Therefore, the correctness of the method used is verified theoretically and practically.
\end{abstract}

Keywords: High Dimensional Data, Partially Linear Additive Model, Modal Regression, Variable Selection, Adoptive Bridge, B-spline

\section{Introduction}

Variable selection of model is one of the hot topics in modern statistics. With the progress of science and technology continuously, statisticians need to process large scale data and select valuable information from these large scale data for statistical analysis.In modern scientific research and technological development, the emergence of high dimensional data has brought new challenges to statisticians. The emergence of high-dimensional data and new scientific problems have changed the ideas of traditional statistics and data analysis.Reducing dimension and screening characteristic variable have become the primary tasks for high dimensional statistical problems. The large scale calculations brought by the reducing dimension and screening characteristic variable process will also promote the continuous improvement of the algorithm.

The partially linear additive model is proposed by Hastie and Tibshirani [1] combining the basic additive model and the characteristics of the partially linear model.It can be defined as follows:

$$
Y_{i}=X_{i}^{T} \beta_{n}+\sum_{l=1}^{d_{n}} g_{l}\left(Z_{l}\right)+\varepsilon_{i}
$$

where $Y$ is response variable, $X=\left(X_{1}{ }^{T}, X_{2}{ }^{T}, \cdots, X_{p_{n}}{ }^{T}\right)$ and $Z=\left(Z_{1}^{T}, Z_{2}^{T}, \cdots Z_{d_{n}}{ }^{T}\right)$ are two group of covariates, $g_{1}\left(Z_{l}\right), \cdots, g_{d_{n}}\left(Z_{l}\right)$ is $d_{n}$-dimensional vector of additive, $\beta=\left(\beta_{1}, \cdots, \beta_{d_{n}}\right)$ is $p_{n}$-dimensional vector of unknown regression coefficient, $\varepsilon$ is model error, which is independent of $X, Z$, and $E(\varepsilon \mid X)=0$.The dimensions $p_{n}$ and $d_{n}$ in the article increase with the increase of the sample size, that is, the sizes of $p_{n}$ and $d_{n}$ are related to that of $n$.

A large number of scholars have studied the partially linear model.Guo [2] proposed an estimation method based on compound quantile regression for semi-parametric partially linear additive model.Liu [3] studied the asymptotic normality of the parameter estimation, used the SCAD penalty function to identify important linear components, it proved that the estimation of non-zero components has Oracle properties.Xia 
[4] focus on the variable selection for semiparametric model with response missing at random.Fan and Huang [5] used profile least squares to estimate the variables of the parameter part, discussed the estimation with asymptotic properties, and tested the model by the Profile generalized likelihood ratio method.Hoshino [6] studied the estimation problem for partially linear additive quantile regression model.

In the high-dimensional model, Meinshausen and Bhlmann [7] studies High-dimensional graphs and variable selection with the lasso.Zhang and Huang [8] discussed The sparsity and bias of the lasso selection in high-dimensional linear regression.Using the bridge penalty method, Wang et al. [9] studies variable selection and parameter estimation in the partially linear model and high-dimensional generalized linear models.Li et al.[10] discussed the variable selection of the generalized semi-varying coefficient model in the ultrahigh-dimensional case.Inspired by the above literature, this paper considers the robust estimation and variable selection for partially linear additive model based on the method of modal regression and adoptive bridge estimation under high dimensional data.It is proved by theoretical properties that adoptive bridge estimation can accurately screen nonzero parameters with probability tending to 1 under highdimensional data.

\section{Variable Selection Method}

Similar to the reference [11], the basis function approximation is used to replace the additive function in (1).

Let

$$
B(U)=\left(B_{1}(U), \cdots, B q_{n}(U)\right)^{T}
$$

be B-spline basic function with the order of $m+1$ where $q_{n}=K+m+1$ and $K$ is the number of interior knot.In order to obtain the consistent estimation of $\left\{g_{l}\left(Z_{l}\right)\right\}$, we use empirically centralized B-spline function subspace

$$
S_{l}^{0}=\left\{s \mid s=\sum_{j=1}^{q_{n}} B_{j}\left(z_{l}\right)^{T} \gamma_{l j}, \sum_{i=1}^{n} s\left(Z_{i l}\right)=0\right\},
$$

its empirically centralized basis function is

$$
\psi_{l j}\left(z_{l}\right)=B_{j}\left(z_{l}\right)-\sum_{i=1}^{n} B_{j}\left(Z_{i l}\right) / n, j=1, \cdots, q_{n},
$$

then

$$
g_{l}\left(z_{l}\right) \approx \sum_{l=1}^{q_{n}} B_{j}\left(z_{l}\right)^{T} \gamma_{l j}, l=1,2, \cdots, d_{n} . g_{l}\left(Z_{l}\right)
$$

can be approximated by

$$
\begin{aligned}
g_{l}\left(z_{l}\right) \approx \sum_{j=1}^{q_{n}} \gamma_{l j} \psi_{l j}\left(z_{l}\right)=\psi_{l}^{T} \gamma_{l}, & \begin{array}{l}
\text { proposed by Fan [13] to approximate the pen } \\
\tilde{\theta}_{n}^{(0)} \text { is very close to 0, let } \tilde{\theta}_{n}^{(0)}=0, \text { otherwise }
\end{array} \\
& \lambda_{1 k} w_{k}\left|\beta_{n k}\right|^{\zeta} \approx \lambda_{1 k} w_{k}\left|\beta_{n k}^{(0)}\right|^{\zeta}+\frac{1}{2}\left\{\frac{\lambda_{1 k} w_{k} \zeta\left|\beta_{n k}^{(0)}\right|^{\zeta-1}}{\left|\beta_{n k}^{(0)}\right|}\right\}\left(\left|\beta_{n k}\right|^{2}-\left|\beta_{n k}^{(0)}\right|^{2}\right),
\end{aligned}
$$

where $\psi_{l}\left(z_{l}\right)=\left(\psi_{l 1}\left(z_{l}\right), \cdots, \psi_{l q_{n}}\left(z_{l}\right)\right)^{T}$ and $\gamma_{l}=$ $\left(\gamma_{l 1}, \gamma_{l 2}, \cdots, \gamma_{l q_{n}}\right)^{T}$ are B-spline coefficients.Denote $\psi_{i l}=\left(\psi_{l l}\left(Z_{i l}\right), \cdots, \psi_{l q}\left(Z_{i l}\right)\right)^{T}$ and $\Psi_{i}=$ $\left(\psi_{i 1}^{T}, \cdots, \psi_{i d_{n}}^{T}\right)^{T}$.Using the modal regression method proposed by Yao [12], we obtain the estimation $\hat{\beta_{n}}$ and $\hat{\gamma_{n}}$ of $\beta_{n}$ and $\gamma_{n}$ by maximizing equation (1).

$$
Q_{h}\left(\gamma_{n}, \beta_{n}\right)=\sum_{i=1}^{n} \phi_{h}\left(Y_{i}-X_{i}^{T} \beta_{n}-\Psi_{i}^{T} \gamma_{n}\right)
$$

where $\phi_{h}(t)$ equals $h^{-1} \phi(t / h), \quad h$ is bandwidth which plays the role of robust estimation, and $\phi(t)$ is a kern density function.In order to calculate and discuss properties conveniently, in this paper we use the normal kernel density function, that is, $\phi_{h}(t)=\frac{1}{\sqrt{2 \pi} h} e^{-\frac{t^{2}}{2 h^{2}}}$. Consider the following penalty function based on equation (1), we have

$$
\begin{aligned}
P_{n}\left(\gamma_{n}, \beta_{n}\right) & =Q_{h}\left(\gamma_{n}, \beta_{n}\right)-\lambda_{1 k} w_{k} \sum_{k=1}^{p_{n}}\left|\beta_{n k}\right|^{\zeta} \\
& -\lambda_{2 l} w_{l} \sum_{1=1}^{d_{n}}\left\|\gamma_{n l}\right\|_{H}{ }^{\zeta}
\end{aligned}
$$

where

$$
\left\|\gamma_{n l}\right\|_{H}=\sqrt{\gamma_{n l}^{T} H \gamma_{n l}}, H=\int{ }_{0}^{1} \psi_{l k}(z) \psi_{l k^{\prime}}(z) d z,
$$

$\lambda_{1 k}$ and $\lambda_{2 l}$ are tuning parameters more than $0 . w_{k}$ and $w_{l}$ are the penalty weights for the k-th and 1-th components, respectively.We default $w_{k}=\left|\tilde{\beta}_{n k}\right|^{-r}$ and $w_{l}=\left|\tilde{\gamma}_{n l}\right|^{-r}$ generally, $\tilde{\gamma}_{n l}$ and $\tilde{\beta}_{n k}$ are non-penalty estimation of $\gamma_{n l}$ and $\beta_{n l}$, respectively. $0<\zeta<1$, the definition of the adoptive bridge estimation is as follows:

$$
\hat{\theta}_{n}=\left(\hat{\gamma}_{n}, \hat{\beta}_{n}\right)=\underset{\hat{\gamma}_{n}, \hat{\beta}_{n}}{\arg \max }\left\{P_{n}\left(\gamma_{n}, \beta_{n}\right)\right\}
$$

It is more difficult to maximize the objective function (2) for a given tuning parameter $\lambda_{1 k}$ and $\lambda_{2 l}$ directly.Assuming that the initial value $\tilde{\theta}_{n 0}=\left(\tilde{\beta}_{n 0}, \tilde{\gamma}_{n 0}\right)^{T}$ is very close to the maximum value of the objective function (2), the initial value is usually considered to be the non-penalty estimation of equation (2). We use the local quadratic algorithm (LQA) proposed by Fan [13] to approximate the penalty function. If 


$$
\lambda_{2 l} w_{l}\left\|\gamma_{n l}\right\|_{H}{ }^{\zeta} \approx \lambda_{2 l} w_{l}\left\|\gamma_{n l}^{(0)}\right\|_{H}{ }^{\zeta}+\frac{1}{2}\left\{\frac{\lambda_{2 l} w_{l} \zeta\left\|\gamma_{n l}^{(0)}\right\|_{H}{ }^{\zeta-1}}{\left\|\gamma_{n l}^{(0)}\right\|_{H}}\right\}\left(\gamma_{n l}^{T} H \gamma_{n l}-\gamma_{n l}^{(0)}{ }^{T} H \gamma_{n l}^{(0)}\right) .
$$

Denote

$$
\begin{gathered}
G(x, z, h)=E\left\{\phi^{\prime}{ }_{h}(\varepsilon)^{2} \mid X=x, Z=z\right\}, \quad F(x, z, h)=E\left\{\phi^{\prime \prime}{ }_{h}(\varepsilon) \mid X=x, Z=z\right\}, \\
a_{n}=\max _{k, l}\left\{\lambda_{1 k}, \lambda_{2 l}: k=1,2, \cdots, s_{1} ; l=1,2, \cdots, s_{2}\right\}, \quad b_{n}=\min _{k, l}\left\{\lambda_{1 k}, \lambda_{2 l}: k=1,2, \cdots, s_{1} ; l=1,2, \cdots, s_{2}\right\} .
\end{gathered}
$$

\section{Asymptotic Properties of Variable Selection}

Let $H_{r}$ represent the whole of function $h(t)$ that satisfies certain conditions in interval $[0,1], h^{m}(t)$ is $m$-th derivative of $h(t)$, it is continuous and satisfies $v$-th order condition of Hölder, $r=m+v$.That is, there is a constant value $M_{0} \in(0, \infty)$ make $\left|h^{m}(s)-h^{m}(t)\right| \leq M_{0}|s-t|^{v}$ is true, where $s, t \in[0,1]$.In order to prove the conclusion of the theorem, the following regular conditions are needed in this paper.

(B1) $E\left(g_{l}\left(Z_{l}\right)\right)=0$ and $g_{l}\left(z_{l}\right) \in H_{r}, l=1, \cdots, d_{n}$, $r>1 / 2$.

(B2) Covariates $Z_{l}$ is a continuous density function $f_{z_{l}}\left(z_{l}\right)$, constants $\delta_{1}$ and $\delta_{2}$ enables $f_{z_{l}}\left(z_{l}\right)$ to satisfy $0<$ $\delta_{1} \leq f_{z_{l}}\left(z_{l}\right) \leq \delta_{2}<\infty$ on interval $[0,1]$.

(B3) Random variables $X_{i k}$ and the eigenvalues of $E\left\{X_{i} X_{i}^{T} \mid Z_{i}\right\}$ are uniformly bounded, where $1 \leq i \leq n$, $1 \leq k \leq p_{n}$.

(B4) Let $t_{1}, \cdots, t_{K}$ be the interior knots of $[0,1]$. Moreover, let $t_{0}=0, t_{K+1}=1, \xi_{i}=t_{i}-t_{i-1}$ and $\xi=\max \left\{\xi_{i}\right\}$.Then, there exists a constant $C_{0}$ such that

$$
\frac{\xi}{\min \left\{\xi_{i}\right\}} \leq C_{0}, \quad \max \left\{\left|\xi_{i+1}-\xi_{i}\right|\right\}=o\left(K^{-1}\right) .
$$

(B5) Let $F(x, z, h)$ and $G(x, z, h)$ are continuous with respect to $(x, z)$.In addition, $F(x, z, h)<0, \forall h>0$.

(B6) $E\left(\phi_{h}^{\prime}(\varepsilon) \mid x, z\right)=0, \quad E\left(\phi^{\prime \prime}{ }_{h}(\varepsilon)^{2} \mid x, z\right)$, $E\left(\phi_{h}^{\prime}(\varepsilon)^{3} \mid x, z\right)$ and $E\left(\phi^{\prime \prime \prime}{ }_{h}(\varepsilon) \mid x, z\right)$ are continuous with respect to $(x, z)$.

(B7) $\lambda_{n} w_{n} s_{n}{ }^{-1 / 2} \rightarrow 0$, where $\lambda_{n}$ is $\lambda_{1 k}$ or $\lambda_{2 l}, \omega_{n}$ is $\omega_{1 k}$ or $\omega_{2 l}$ and $s_{n}$ is $s_{1}$ or $s_{2}$.

(B8) For $a_{n}$ and $b_{n}$, there are $\sqrt{n} a_{n} \rightarrow 0$ and $\sqrt{n} b_{n} \rightarrow \infty$ where $n \rightarrow \infty$.

Remark 1 Conditions (B1)-(B3) are common conditions for partially linear additive model; Condition (B4) indicates that $c_{0}, \cdots, c_{K+1}$ is a $C_{0}$-quasi-uniform sequence of partitions of $[0,1]$; Conditions (B5)-(B6) are required for the modal regression.Reference Yao[12] thought $E\left(\phi_{h}^{\prime}(\varepsilon) \mid x, z\right)=$
0 guarantees the consistency of the estimation.If the error distribution density is symmetrical about 0 , these conditions are automatically satisfied; Condition (B7) is an indispensable condition for the adoptive bridge estimation of convergence and oracle properties; Condition (B8) represents the assumption of adoptive bridge estimation.

Now, we discuss the asymptotic properties of penalty estimation $\hat{\beta_{n}}$ and $\hat{\gamma_{n}}$. Let $g_{l}{ }^{(0)}\left(z_{l}\right)$ and $\beta_{n}{ }^{(0)}$ be the real value of $g_{l}\left(z_{l}\right)$ and $\beta_{n}$.Default $\beta_{n k}{ }^{(0)}=0$ can represent an unrelated variable, where $k=s_{1}+1, \cdots, p_{n}, \beta_{n k}{ }^{(0)} \neq 0$ can represent a function corresponding to an important variables where $k=$ $1,2, \cdots s_{1}$. Similarly, we have $g_{l}{ }^{(0)}\left(z_{l}\right)=0$ and $g_{l}{ }^{(0)}\left(z_{l}\right) \neq 0$ where $l=s_{2}+1, \cdots, d_{n}$ and $l=1,2, \cdots s_{2}$, respectively.

First, we give the consistency of the penalty estimations.

Theorem 1 Suppose that the regular conditions (B1)-(B6) hold, the number of knots $K=O\left(n^{1 /(2 r+1)}\right), 0<\zeta<1$, then we have

(i) $\left\|\hat{\beta}_{n k}-\beta_{n k}^{(0)}\right\|=O_{p}\left(n^{-r / 2 r+1}+a_{n}\right)$,

(ii) $\left\|\hat{g}_{l}\left(z_{l}\right)-g_{l}{ }^{(0)}\left(z_{l}\right)\right\|=O_{p}\left(n^{-r / 2 r+1}+a_{n}\right)$.

Next, we can obtain the sparsity of the penalty estimation under certain conditions.

Theorem 2 Suppose that the regular conditions (B1)-(B6) hold, $0<\zeta<1$, then we have

(i) $\hat{\beta}_{n k}=0, k=s_{1}+1, \cdots, p_{n}$,

(ii) $\hat{g}_{l}=0, l=s_{2}+1, \cdots, d_{n}$.

Denote

$$
\beta_{n 1}=\left(\beta_{n 1}^{T}, \cdots, \beta_{n s_{1}}^{T}\right)^{T}, \gamma_{n 1}=\left(\gamma_{n 1}^{T}, \cdots, \gamma_{n s_{2}}^{T}\right)^{T},
$$

and let $\beta_{n 1}{ }^{(0)}$ and $\gamma_{n 1}{ }^{(0)}$ be the real true of $\beta_{n 1}$ and $\gamma_{n 1}$ respectively. Their respective corresponding covariates are labeled with $X_{1}$ and $\Psi_{1}$. In addition, define

$$
\begin{aligned}
& \Upsilon=E\left(\phi^{\prime \prime}{ }_{h}(\varepsilon) \Psi_{1} \Psi_{1}^{T}\right)=E\left(F(x, z, h) \Psi_{1} \Psi_{1}^{T}\right) \\
& \Omega=E\left(\phi^{\prime \prime}{ }_{h}(\varepsilon) \Psi_{1} X_{1}^{T}\right)=E\left(F(x, z, h) \Psi_{1} X_{1}^{T}\right) .
\end{aligned}
$$

Finally, we give the asymptotic normality of the non-zero components in parameters.

Theorem 3 Under the conditions of Theorem 2 and (B1)-(B8), we have

$$
\sqrt{n}\left(\hat{\beta}_{n 1}-\beta_{n 1}^{(0)}\right) \stackrel{D}{\rightarrow} N\left(0, \sum^{-1} \Gamma \sum^{-1}\right),
$$


where $\Gamma$ is $E\left(G(X, Z, h) \breve{X}_{1} \breve{X}_{1}^{T}\right), \Sigma$ is $E\left(F(X, Z, h) \breve{X}_{1} \breve{X}_{1}^{T}\right)$ and $\breve{X}_{1}$ is $X_{1}-\Omega^{T} \Upsilon^{-1} \Psi_{1}$.

\section{Estimation Algorithm and Parameter Selection}

\subsection{Estimation Algorithm}

The following is the algorithm which has combined EM algorithm with LQA algorithm, it is suitable for this paper.Assume that the previous estimations are $\hat{\gamma}_{n l}^{(m)}$ and $\hat{\beta}_{n k}^{(m)}$ respectively, where $l=1,2, \cdots, p_{n}$ and $k=1,2, \cdots d_{n}$.If they are very close to 0 , then let $\hat{\gamma}_{n j}^{(m+1)}=0$ and $\hat{\beta}_{n k}^{(m+1)}=0$; otherwise, iterative estimation is performed according to the following algorithm steps.

Denote

and let

$$
Z_{i}^{T}=\left(X_{i}^{T}, \Psi_{i}^{T}\right)^{T}, \theta_{n}=\left(\beta_{n}, \gamma_{n}\right)^{T}
$$

$$
\Sigma_{\lambda_{1}, \lambda_{2}}\left(\theta_{n}^{(m)}\right)=\operatorname{diag}\left\{\lambda_{11} w_{1} \zeta\left\|\gamma_{n 1}^{(0)}\right\|_{H}^{\zeta-1} H, \cdots, \lambda_{1 d_{n}} w_{d_{n}} \zeta\left\|\gamma_{n d_{n}}^{(0)}\right\|_{H}^{\zeta-1} H, \lambda_{21} w_{1} \zeta\left|\beta_{n 1}{ }^{(0)}\right|^{\zeta-1}, \cdots, \lambda_{2 p_{n}} w_{p_{n}} \zeta\left|\beta_{n p_{n}}{ }^{(0)}\right|^{\zeta-1}\right\} .
$$

Based on the EM algorithm, we can obtain the penalty estimation of (3) as follows:

Step 1(E-step): First, the non-penalty estimation in (3) is the initial value $\tilde{\theta}_{n}^{(0)}=\left(\tilde{\beta}_{n}^{(0)}, \tilde{\gamma}_{n}^{(0)}\right)^{T}$ and the termination error, $0<\varepsilon<10^{-6}$.Then let $m=0$ and update $\pi\left(i \mid \theta_{n}{ }^{(m)}\right)$ by

$$
\pi\left(i \mid \theta_{n}^{(m)}\right) \propto \sum_{i=1}^{n} \phi_{h}\left(Y_{i}-Z_{i}^{T} \theta_{n}^{(m)}\right),
$$

where

$$
\begin{aligned}
\tilde{\theta}_{n}^{(0)} & =\arg \max _{\theta} \Sigma_{i=1}^{n}\left\{\pi\left(i \mid \theta_{n}^{(m)}\right) \log \phi_{h}\left(Y_{i}-Z_{i}^{* T} \theta_{n}^{(m)}\right)\right\} \\
& =\left(Z_{i}^{* T} D Z_{i}^{*}\right)^{-1} Z_{i}^{* T} D Y_{i},
\end{aligned}
$$

$D$ is a diagonal matrix of $n \times n$ whose elements of the $i$-th diagonal are $\pi\left(i \mid \theta_{n}{ }^{(m)}\right)$.

Due to

$$
\tilde{\theta}_{n}^{(0)}=\arg \max _{\theta} \sum_{i=1}^{n}\left\{\pi\left(i \mid \theta_{n}^{(m)}\right) \log \phi_{h}\left(Y_{i}-Z_{i}^{* T} \theta_{n}^{(m)}\right)\right\},
$$

and

$$
\phi_{h}(t)=\frac{1}{\sqrt{2 \pi} h} e^{-\frac{t^{2}}{2 h^{2}}} .
$$

By calculating the logarithmic function, we have the target function

$$
Q=\Sigma_{i=1}^{n}\left\{\pi\left(i \mid \theta_{n}^{(m)}\right)\left(Y_{i}-Z_{i}^{* T} \theta_{n}^{(m)}\right)^{2}\right\},
$$

Next, we maximize the target function by calculating partial derivatives, we have

$$
\begin{aligned}
\tilde{\theta}_{n}^{(0)} & =\arg \max _{\theta} \Sigma_{i=1}^{n}\left\{\pi\left(i \mid \theta_{n}^{(m)}\right) \log \phi_{h}\left(Y_{i}-Z_{i}^{* T} \theta_{n}^{(m)}\right)\right\} \\
& =\left(Z_{i}^{* T} D Z_{i}^{*}\right)^{-1} Z_{i}^{* T} D Y_{i} .
\end{aligned}
$$

Step 2(M-step): Update $\hat{\theta}_{n}^{(m)}$ by

$$
\begin{aligned}
\hat{\theta}_{n}^{(m+1)} & =\arg \max \sum_{i=1}^{n}\left\{\pi\left(i \mid \theta_{n}^{(m)}\right) \log \phi_{h}\left(Y_{i}-Z_{i}^{T} \theta_{n}^{(m)}\right)\right\} \\
& +\frac{n}{2} \theta_{n}^{(m)^{T}} \sum_{\lambda_{1}, \lambda_{2}}\left(\theta_{n}^{(m)}\right) \theta_{n}^{(m)} \\
& \approx\left(Z_{i}^{T} D Z_{i}+n \sum_{\lambda_{1}, \lambda_{2}}\left(\theta_{n}^{(m)}\right)\right)^{-1} Z_{i}^{T} D Y_{i} .
\end{aligned}
$$

Step 3: Repeat Step 1 and Step 2 continuously until the algorithms are convergent and the final estimation $\hat{\theta}_{n}=$ $\left(\hat{\beta}_{n}, \hat{\gamma}_{n}\right)$, where $\hat{\beta}_{n}=\left(I_{p_{n} \times p_{n}}, 0_{p_{n} \times d_{n} q}\right) \hat{\theta}_{n}$ and $\hat{\gamma}_{n}=$ $\left(0_{d_{n} q \times p_{n}}, I_{d_{n} q \times d_{n} q}\right) \hat{\theta}_{n}$ is obtained.

\subsection{The Selection of Bandwidth, Node and Tuning Parametet:2.1. Bandwidth Selection}

In order to select the bandwidth handily, we assume that $X$ and $Z$ are independent of each other.On the basis of the asymptotic variance obtained by Theorem 3 and the variance of the Least square B-spline estimation proposed by Zhao [14], we can get the ratio of two asymptotic variances as

$$
r(h)=\frac{G(h) F^{-2}(h)}{\sigma^{2}},
$$

where

$$
\sigma^{2}=E\left(\varepsilon^{2}\right), G(h)=E\left\{\phi_{h}{ }_{h}(\varepsilon)\right\}^{2}, F(h)=E\left\{\phi^{\prime \prime}{ }_{h}(\varepsilon)\right\} .
$$

Define the optimal bandwidth as follows:

$$
h_{\text {opt }}=\operatorname{argmin}_{h} r(h)=\operatorname{argmin} G(H) F^{-2}(H) .
$$

In practical application, since the distribution of errors are unknown, the bandwidth obtained by equation (5) cannot be used directly.A feasible method is to estimate $F(h)$ and $G(h)$ 
by

$$
\hat{F}(h)=\frac{1}{n} \sum_{i=1}^{n} \phi_{h}^{\prime \prime}\left(\hat{\varepsilon}_{i}\right), \hat{G}(h)=\frac{1}{n} \sum_{i=1}^{n}\left\{\phi_{h}^{\prime \prime}\left(\hat{\varepsilon}_{i}\right)\right\}^{2} .
$$

Then we can obtain the estimation of $r(h)$ by

$$
\hat{r}(h)=\hat{G}(h) \hat{F}^{-2}(h) / \hat{\sigma}^{2},
$$

where

$$
\hat{\varepsilon}_{i}=Y_{i}-X_{i}^{T} \hat{\beta}_{n}-\sum_{l=1}^{d_{n}} \hat{g}_{l}\left(z_{l}\right),
$$

$\hat{g}_{l}\left(z_{l}\right)$ and $\hat{\beta}_{n}$ are initial estimations based on the robust method.

In practical, after getting the estimation of $r(h)$, we use the lattice point search method to obtain the $h_{\text {opt }}$.According to the suggestion in reference by Yao [12], some of the possible lattice shops can be taken as $h_{\text {opt }}=0.5 \hat{\sigma} \times 1.02^{j}$, where $j=0,1, \cdots, k, k$ is 50 or 100 .

\subsubsection{The Selection of Node K and Tuning Parameter}

In order to implement the estimation algorithm handily, it is necessary to select the appropriate number of internal nodes $K$ and the tuning parameters $\lambda_{1 k}$ and $\lambda_{2 l}$ in the adoptive bridge penalty estimation. Denote

$$
\lambda_{1 k}=\frac{\lambda}{\left|\tilde{\beta}_{n k}\right|}, \lambda_{2 l}=\frac{\lambda}{\left\|\tilde{\gamma}_{n l}\right\|}
$$

where $\tilde{\gamma}_{n l}$ and $\tilde{\beta}_{n k}$ are non-penalty estimations of $\gamma_{n l}$ and $\beta_{n k}$ respectively. $\lambda$ and $K$ are selected by $\mathrm{CV}$ criterion:

$$
C V(K, \lambda)=\sum_{i=1}^{n} \phi_{h}\left[Y_{i}-\Psi_{i}^{T} \hat{\gamma}_{n}^{(-i)}-X_{i}^{T} \hat{\beta}_{n}^{(-i)}\right]
$$

where $\hat{\gamma}_{n}^{(-i)}$ and $\hat{\beta}_{n}^{(-i)}$ are penalty estimations obtained by excepting the $i$-th data from equation (4).The optimal node $K_{\text {opt }}$ and tuning parameter $\lambda_{\text {opt }}$ are obtained by

$$
\left(K_{\text {opt }}, \lambda_{\text {opt }}\right)=\max _{K, \lambda} C V(K, \lambda) .
$$

\section{Numerical Simulations}

We simulate data from model (1), where $g_{l}\left(z_{l}\right)$ are the $d_{n^{-}}$ dimensional vectors, $g_{1}\left(Z_{i 1}\right)=2 Z_{i 1}-E\left(2 Z_{i 1}\right), g_{2}\left(Z_{i 2}\right)=$ $\left(3 Z_{i 2}-1\right)^{2}-E\left(\left(3 Z_{i 2}-1\right)\right)$ and $g_{3}\left(Z_{i 3}\right)=4 \sin \left(4 \pi Z_{i 3}\right)-$ $E\left(4 \sin \left(4 \pi Z_{i 3}\right)\right)$, the rest of $g_{l}\left(z_{l}\right)$ are zero; $\beta_{n}$ is $p_{n}$ dimensional vector, $\beta_{1}=1.8, \beta_{2}=1, \beta_{3}=0.5, \beta_{4}=-0.8$, $\beta_{5}=-0.3$, the rest of $\beta_{n}$ are zero.In this paper, both $p_{n}$ and $d_{n}$ increase as the sample size $n$ increases gradually, so we assume $p_{n}=d_{n}$.To perform this simulation, we take the covariates $X \sim N(0,1)$ and $Z \sim U(0,1)$.The model error $\varepsilon_{i}$ is subject to the three cases of $N(0,1), t(4)$ and $0.25 N(0,6)+0.75 N(0,1)$ respectively.The simulated sample size $n$ is taken as 100, 200 and 400 respectively.And the corresponding $p_{n}$ is taken as 10,20 and 40 .Repeat 400 times for each simulation and calculate its average.We use a third order B-spline basic function to approximate the function of the variable coefficient part in the simulation, and optimal node and tuning parameters are selected by $\mathrm{CV}$ method.In order to test the effect of variable selection under high-dimensional data, we also calculate the penalty estimations through LASSO and adoptive bridge estimation based on the modal regression in this paper.We consider the cases of $\zeta=0.6$ and $\zeta=0.85$ in the adoptive bridge estimation.

To illustrate the proposed method in this paper, the efficient estimations $\hat{g}_{l}\left(z_{l}\right)$ will be assessed by using the square root of average square errors $(R A S E)$

$$
R A S E=\left\{n^{-1} \sum_{l=1}^{\text {grid }}\left\|\hat{g}_{l}\left(z_{l}\right)-g_{l}\left(z_{l}\right)\right\|^{2}\right\}^{1 / 2} .
$$

The generalized mean square error $(G M S E)$ is used to evaluate the performance of $\hat{\beta}_{n}$ GMSE $=$ $\left(\hat{\beta}_{k}-\beta_{k}\right) E\left(X X^{T}\right)\left(\hat{\beta}_{k}-\beta_{k}\right) . \mathrm{CN}$ and $\mathrm{CP}$ are used to represent the number of important variables for correctly estimating additive coefficients and parameters respectively.

It can be seen from Table (1) that when the error obeys standard normal distribution, $\mathrm{t}$-distribution or mixed normal distribution, the values of MRSE and GMSE under adoptive bridge estimation method are generally smaller than those under LASSO.This conclusion is still true when the sample size and dimension are getting larger, and as the dimension and sample size increase, the estimation accuracy of the model also increases.If the dimension and sample size are fixed, the values of RASE and GMSE when the error obeys the normal distribution are smaller than those when the error obeys tdistribution, which indicates that the simulation effect is better under the normal distribution.

\section{Proof of Theorems}

Proof of Theorem 1 Let $\delta=n^{-r / 2 r+1}+a_{n}$ and $v=$ $\left(v_{1}^{T}, v_{2}^{T}\right)^{T}$, where $v_{1}^{T}$ is a $p_{n}$-dimensional vector, $v_{2}^{T}$ is a $d_{n}$ dimensional vector.Denote $\beta_{n}=\beta_{n}{ }^{(0)}+\delta v_{1}$ and $\gamma_{n}=\gamma_{n}{ }^{\left({ }^{0}\right)}+$ $\delta v_{2}$.For part (i), we first prove for any given $v_{1}^{T}$ and $v_{2}^{T}$, there exists

$$
\operatorname{Pr}\left\{\sup P_{n}\left(\gamma_{n}, \beta_{n}\right)-P_{n}\left(\gamma_{n}{ }^{(0)}, \beta_{n}{ }^{(0)}\right)>0\right\} \rightarrow 1,
$$

Define 


$$
\begin{aligned}
\Pi\left(\gamma_{n}, \beta_{n}\right)= & \frac{1}{K}\left\{P_{n}\left(\gamma_{n}, \beta_{n}\right)-P\left(\gamma_{n}{ }^{(0)}, \beta_{n}{ }^{(0)}\right)\right\} \\
= & -\frac{-\delta}{K} \sum_{i=1}^{n} \phi^{\prime}{ }_{h}\left(\varepsilon_{i}+1_{d_{n}}^{T} R\left(Z_{i}\right)\right)\left(X_{i}^{T} v_{1}+\Psi_{i}^{T} v_{2}\right)+\frac{\delta^{2}}{K} \sum_{i=1}^{n} \phi^{\prime \prime}{ }_{h}\left(\varepsilon_{i}+1_{d_{n}}^{T} R\left(Z_{i}\right)\right)\left(X_{i}^{T} v_{1}+\Psi_{i}^{T} v_{2}\right)^{2} \\
& +\frac{\delta^{3}}{K} \sum_{i=1}^{n} \phi^{\prime \prime}{ }_{h}\left(\varepsilon_{i}^{*}\right)\left(X_{i}^{T} v_{1}+\Psi_{i}^{T} v_{2}\right)^{3} \\
& +\frac{n}{K} \lambda_{1 l} w_{l} \sum_{l=1}^{d_{n}}\left\{\left\|\gamma_{n l}\right\|_{H}^{\zeta}-\left\|\gamma_{n l}(0)\right\|_{H}^{\zeta}\right\}+\frac{n}{K} \lambda_{2 k} w_{k} \sum_{k=1}^{p_{n}}\left\{\left|\beta_{n k}\right|^{\zeta}-\left|\beta_{n k}{ }^{(0)}\right|^{\zeta}\right\} \\
\triangleq & I_{n 1}+I_{n 2}+I_{n 3}+I_{n 4}+I_{n 5},
\end{aligned}
$$

where $\varepsilon_{i}^{*}$ is between $\varepsilon_{i}+1_{d_{n}}^{T} R\left(Z_{i}\right)$ and $\varepsilon_{i}+1_{d_{n}}^{T} R\left(Z_{i}\right)-\delta\left(X_{i}^{T} v_{1}+\Psi_{i}^{T} v_{2}\right)$, and we denote $R(U)=\left(R_{1}(U), \cdots, R_{d_{n}}(U)\right)^{T}$, $R_{l}(Z)=g_{0 l}(Z)-\psi_{l}^{T} \gamma_{0 l}$ and $l=1,2, \cdots, d_{n}$. According to the regular conditions (B1) and (B2) and the conclusions in Schumaker [15], there exists $\left\|R_{l}\left(Z_{i}\right)\right\|=O\left(K^{-r}\right)$. We get $E\left(\phi_{h}{ }^{\prime}\left(\varepsilon_{i}\right) \mid x, z, u\right)=0$ by expanding $I_{n 1}$ by Taylor formula and regular conditions (B4) and (B6).By simple calculation, we get

$$
\begin{aligned}
I_{n 1} & =-K^{-1} \delta \sum_{i=1}^{n} \phi_{h}^{\prime}\left(\varepsilon_{i}+1_{d_{n}}^{T} R\left(Z_{i}\right)\right)\left(X_{i}^{T} v_{1}+\Psi_{i}^{T} v_{2}\right) \\
& =O_{p}\left(n K^{-r}\right)\|v\| .
\end{aligned}
$$

Similarly, we get $I_{n 1}=o_{p}\left(I_{n 2}\right)$ and $I_{n 3}=o_{p}\left(I_{n 2}\right)$. For $I_{n 5}$, by using the inequality Hölder, we get

$$
\left|I_{n 5}\right| \leq C_{2} \lambda_{2} w_{k} \zeta p_{n}{ }^{-1}\left(\sum_{k=1}^{p_{n}} p_{n}{ }^{2}\right)^{1 / 2}\left(\sum_{k=1}^{p_{n}}\left(\beta_{n k}-\beta_{n k}(0)\right)^{2}\right)^{1 / 2}, k=1,2, \cdots, s_{2} .
$$

According to the regular condition (B7) we have $\left|I_{n 5}\right| \leq C_{2} \lambda_{2} w_{k} \zeta p_{n}^{-1 / 2} \delta\|v\| \rightarrow 0$. That is, $I_{n 5}$ is consistently controlled by $I_{n 2}$. Similarly, $I_{n 4}$ is consistently controlled by $I_{n 2}$.

In summary, according to the regular condition (B5) $F(x, z, h)<0$, so if we select a sufficiently large C and a vector of $\|v\|$ that can satisfy the condition, $\Pi_{n}\left(\gamma_{n}, \beta_{n}\right)<0$, which means

$$
\operatorname{Pr}\left\{\sup P_{n}\left(\gamma_{n}, \beta_{n}\right)-P_{n}\left(\gamma_{n}^{(0)}, \beta_{n}^{(0)}\right)>0\right\} \rightarrow 1
$$

Thus, there is a local maximum point that satisfies $\left\|\hat{\beta}_{n k}-\beta_{n k}{ }^{(0)}\right\|=O_{p}\left(n^{-r / 2 r+1}+a_{n}\right)$. That is, part (i) is proven. We use the similar method in reference [12] to complete the second part of the proof.

Proof of Theorem 2 First, we prove part (i).According to the conclusion of Theorem 1, for any given sufficiently small $v=$ $C n^{-r /(2 r+1)}$, when $n \rightarrow \infty$, for any $\gamma_{n j}$ that satisfies $\left\|\gamma_{n l}-\gamma_{n l}^{(0)}\right\|=O_{P}\left(n^{\frac{-r}{2 r+1}}\right)$ and $\beta_{n k}$ that satisfies $\left\|\beta_{n k}-\beta_{n k}^{(0)}\right\|=$ $O_{P}\left(n^{\frac{-r}{2 r+1}}\right), k=1, \cdots, s_{1}$, with probability tending to 1 , we have

$$
\begin{gathered}
\frac{\partial P_{n}\left(\gamma_{n}, \beta_{n k}\right)}{\partial \beta_{n k}}<0, \text { if } 0<\beta_{\mathrm{nk}}<\mathrm{v}, \text { where } \mathrm{k}=\mathrm{s}_{1}+1, \mathrm{~s}_{1}+2, \cdots, \mathrm{p}_{\mathrm{n}}, \\
\frac{\partial P_{n}\left(\gamma_{n}, \beta_{n k}\right)}{\partial \beta_{n k}}>0, \text { if }-\mathrm{v}<\beta_{\mathrm{nk}}<0, \text { where } \mathrm{k}=\mathrm{s}_{1}+1, \mathrm{~s}_{1}+2, \cdots, \mathrm{p}_{\mathrm{n}} .
\end{gathered}
$$

Similar to the proof of Theorem 1 , we get

$$
\frac{\partial P_{n}\left(\gamma_{n l}, \beta_{n k}\right)}{\partial \beta_{n k}}=-n \lambda_{2 k} \zeta\left[\left|\beta_{n k}\right|^{\zeta-1} \operatorname{sgn}\left(\beta_{n k}\right)+O_{p}\left(n^{-1} \lambda_{2 k}{ }^{-1} \zeta^{-1} n^{\frac{-r}{2 r+1}}\right)\right] .
$$

By the regular conditions (B3), (B7) and (B8), with probability tending to 1 , we have $\lambda_{2 k} n^{-r / 2 r+1} \geq b_{n} n^{-r / 2 r+1} \rightarrow \infty$ where $k=s_{1}+1, \cdots, p_{n}$. Therefore, the sign of $\partial P_{n}\left(\gamma_{n}, \beta_{n k}\right) / \partial \beta_{n k}$ is completely determined by the sign of $\beta_{n k}$, that is, the equations of (15) and (16) are established. 
For the proof of part (ii), using a method similar to the above, with probability to 1 we have $\hat{g}_{n l}\left(z_{l}\right)=0$, where $l=$ $s_{2}+1, \cdots, d_{n}$, that is, the conclusion of part (ii) is established.

Proof of Theorem 3 From the proof of Theorem 1 and Theorem 2, we know that $P_{n}\left(\gamma_{n}, \beta_{n}\right)$ attains the maximal value at $\left(\hat{\beta}_{n 1}^{T}, 0\right)^{T}$ and $\left(\hat{\gamma}_{n 1}^{T}, 0\right)^{T}$ with probability tending to 1 when $n \rightarrow \infty$. Denote

$$
P_{n 1}\left(\gamma_{n}, \beta_{n}\right)=\frac{\partial P_{n 1}\left(\gamma_{n}, \beta_{n}\right)}{\partial \beta_{n 1}} \text { then }\left(\hat{\beta}_{\mathrm{n} 1}^{\mathrm{T}}, 0\right)^{\mathrm{T}}
$$

must satisfy the following equation

$$
\begin{aligned}
& \frac{1}{n} P_{n 1}\left(\left(\hat{\gamma}_{n 1}^{T}, 0\right)^{T},\left(\hat{\beta}_{n 1}^{T}, 0\right)^{T}\right) \\
& =\frac{1}{n} \sum_{i=1}^{n} X_{i a} \phi_{h}^{\prime}\left(Y_{i}-\Psi_{i}^{T} \hat{\gamma}_{n l}-X_{i}^{T} \hat{\beta}_{n k}\right)-\lambda_{2 k} w_{k} \zeta \sum_{k=1}^{p_{n}}\left|\hat{\beta}_{n k}\right|^{\zeta-1} \operatorname{sgn}\left(\hat{\beta}_{n k}\right)=0 .
\end{aligned}
$$

Expanding the second term below the equation (17) by Taylor formula, we have

$$
\begin{aligned}
& n^{-1} \sum_{i=1}^{n} Z_{i 1}\left\{\phi_{h}^{\prime}\left(\epsilon_{i}\right)+\phi_{h}^{\prime \prime}\left(\epsilon_{i}\right)\left[X_{i}^{T} R^{*}\left(U_{i}\right)-Z_{i 1}^{T}\left(\hat{\beta}_{n 1}-\beta_{n 1}{ }^{(0)}\right)-W_{i 1}^{T}\left(\hat{\gamma}_{n 1}-\gamma_{n 1}{ }^{(0)}\right)\right]\right. \\
& \left.\quad+\phi^{\prime \prime \prime}{ }_{h}\left(\varsigma_{i}\right)\left[X_{i}^{T} R^{*}\left(U_{i}\right)-Z_{i 1}^{T}\left(\hat{\beta}_{n 1}-\beta_{n 1}{ }^{(0)}\right)-W_{i 1}^{T}\left(\hat{\gamma}_{n 1}-\gamma_{n 1}{ }^{(0)}\right)\right]^{2}\right\}+o_{p}\left(\hat{\beta}_{n 1}-\beta_{n 1}{ }^{(0)}\right)=0 .
\end{aligned}
$$

where $\varsigma_{i}$ is between $\varepsilon_{i}$ and $Y_{i}-\Psi_{i 1}^{T} \hat{\gamma}_{n 1}-X_{i 1}^{T} \hat{\beta}_{n 1}, R^{*}(u)=\left(R_{1}(u), \cdots, R_{s_{1}}(u)\right)^{T}$. According to the regular conditions $\sqrt{n} b_{n} \rightarrow \infty$ and $\left(\hat{\beta}_{n k}-\beta_{n k}^{(0)}\right)=o_{p}(1)$, we obtain

$$
\hat{\gamma}_{n 1}-\gamma_{n 1}^{(0)}=\left(\Upsilon_{n}+o_{p}(1)\right)^{-1}\left\{-\Omega_{n}\left(\hat{\beta}_{n 1}-\beta_{n 1}{ }^{(0)}\right)+\mathrm{T}_{n}\right\} .
$$

where

$$
\mathrm{T}_{n}=n^{-1} \sum_{i=1}^{n} W_{i 1}\left[\phi_{h}^{\prime}\left(\varepsilon_{i}\right)+\phi_{h}^{\prime \prime}\left(\varepsilon_{i}\right) X_{i 1}^{T} R^{*}\left(U_{i}\right)\right] .
$$

Substituting into equation (16), we obtain

$$
E\left(n^{-1} \sum_{i=1}^{n} \phi_{h}^{\prime \prime}\left(\varepsilon_{i}\right) \Omega^{T} \Upsilon^{-1} \Psi_{i 1}\left[X_{i 1}^{T}-\Psi_{i 1}^{T} \Upsilon^{-1} \Omega^{T}\right]\right)=0
$$

After calculations, we have

$$
\begin{aligned}
& \left\{n^{-1} \sum_{i=1}^{n} \phi^{\prime \prime}{ }_{h}\left(\epsilon_{i}\right) \breve{Z}_{i 1} \breve{Z}_{i 1}^{T}+o_{p}(1)\right\} \sqrt{n}\left(\hat{\beta}_{n 1}-\beta_{n 10}\right) \\
& =n^{-1 / 2} \sum_{i=1}^{n} \breve{Z}_{i 1} \phi^{\prime}{ }_{h}\left(\varepsilon_{i}\right)+n^{-1 / 2} \sum_{i=1}^{n} \breve{Z}_{i 1} \phi^{\prime \prime}{ }_{h}\left(\varepsilon_{i}\right) X_{i}^{T} R^{*}\left(U_{i}\right)-n^{-1 / 2} \sum_{i=1}^{n} \breve{Z}_{i 1} \phi^{\prime \prime}{ }_{h}\left(\varepsilon_{i}\right)\left(\Upsilon^{-1}+o_{p}(1)\right) \mathrm{T}_{n} \\
& =J_{n 1}+J_{n 2}+J_{n 3},
\end{aligned}
$$

where $\bar{X}_{i 1}=X_{i 1}-\Omega_{n}{ }^{T} \Upsilon_{n}{ }^{-1} \mathrm{~T}_{i}$.According to the definition of $R^{*}\left(U_{i}\right)$, for $J_{n 2}$, we have

$$
\begin{aligned}
J_{n 2} & =n^{-1 / 2} \sum_{i=1}^{n} \phi_{h}^{\prime \prime}\left(\varepsilon_{i}\right)\left(Z_{i}^{T}-E\left(\Omega_{n}\right)^{T} E\left(\Upsilon_{n}\right)^{-1} W_{i 1}\right) X_{i 1}^{T} R^{*}\left(U_{i}\right) \\
& +n^{-1 / 2} \sum_{i=1}^{n} \phi^{\prime \prime}{ }_{h}\left(\varepsilon_{i}\right) E\left(\Omega_{n}\right)^{T}\left(E\left(\Upsilon_{n}\right)^{-1}-\Omega_{n}{ }^{T} \Upsilon_{n}{ }^{-1} W_{i 1}\right) X_{i}^{T} R^{*}\left(U_{i}\right) \\
& =J_{n 21}+J_{n 22} .
\end{aligned}
$$

Since $E\left(\phi^{\prime \prime}{ }_{h}\left(\varepsilon_{i}\right)\left\{X_{i}^{T}-E\left(\Omega_{n}\right)^{T}\left[\left(\Upsilon_{n}\right)^{-1} \Psi_{i 1}{ }^{T}\right] \Psi_{i 1}\right\}\right)=0$, we have

$$
n^{-1 / 2} \sum_{i=1}^{n} \phi_{h}^{\prime \prime}\left(\varepsilon_{i}\right)\left(X_{i}^{T}-E\left(\Omega_{n}\right)^{T} E\left(\Upsilon_{n}\right)^{-1} \Psi_{i 1}^{T}\right) \Psi_{i 1}=o_{p}(1)
$$


Combining $W_{i}=\left(W_{i 1}^{T}, W_{i 2}^{T}, \cdots, W_{i p_{n}}{ }^{T}\right)$ and $\left|R^{*}\left(U_{i}\right)\right|=o_{p}(1)$, we get $J_{n 21}=o_{p}(1)$.Similarly, $J_{n 22}=$ $o_{p}(1)$.

Further, according to the regular conditions, we have

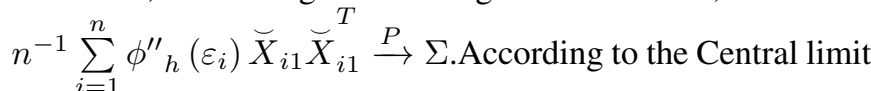
theorem, there is

$$
J_{n 1}=n^{-1 / 2} \sum_{i=1}^{n} \phi_{h}^{\prime}\left(\varepsilon_{i}\right) \breve{X}_{i 1}^{T} \stackrel{d}{\rightarrow} N(0, \Gamma) .
$$

where $\Gamma=E\left(G(x, z, h) \breve{X}_{i 1} \widetilde{X}_{i 1}^{T}\right)$.

For any vectors $\rho$ whose components are not all zero,

$$
\rho^{T} P_{n 1}=n^{-1 / 2} \sum_{i=1}^{n} \rho^{T} \phi_{h}^{\prime}\left(\varepsilon_{i}\right) \breve{X}_{i 1}^{T} \triangleq \sum_{i=1}^{n} o_{i} \xi_{i} .
$$

where $o_{i}{ }^{2}=n^{-1} \sum_{i=1}^{n} G(X, Z, h) \rho^{T} \breve{X}_{i 1} \breve{X}_{i 1}{ }^{T} \rho$.Under the given conditions of $\left\{X_{i}, Z_{i}\right\}, \xi_{i}$ is independent with mean zero and variance one.

Then, we verify Linderberg's central limit theorem.If

$$
\frac{\max o_{i}^{2}}{\sum_{i=1}^{n} o_{i}{ }^{2}} \stackrel{P}{\rightarrow} 0
$$

holds, then

$$
\sum_{i=1}^{n} o_{i} \xi_{i} / \sqrt{\sum_{i=1}^{n} o_{i}{ }^{2}} \stackrel{D}{\rightarrow} N(0,1)
$$

and thus the equation (18) holds.

Next, we prove (19). Owing to $\left(\rho^{T} \breve{Z}_{i 1}^{T}\right)^{2} \leq\|\rho\|^{2}\left\|\breve{Z}_{i 1}\right\|^{2}$, we have $o_{i}{ }^{2} \leq n^{-1} G(X, Z, U, h)\|\rho\|^{2}\left\|\breve{Z}_{i 1}\right\|^{2}$ and $\left\|\breve{Z}_{i 1}\right\|=$ $\left\|Z_{i 1}-\Omega^{T} \Upsilon^{-1} W_{i 1}\right\| \leq\left\|Z_{i 1}\right\|+\left\|\Omega^{T} \Upsilon^{-1} W_{i 1}\right\|$. According to the regular conditions, we have $\max _{i}\left\|Z_{i}\right\| n^{-1 / 2}=o_{p}(1)$ and $\max _{i}\left\|\Omega^{T} \Upsilon^{-1} W_{i}\right\|=o_{p}(1)$. By applying Slutsky theorem again, then we can prove that equation (18) is true.

In conclusion, we obtain

$$
\sqrt{n}\left(\hat{\beta}_{n 1}-\beta_{n 1}{ }^{(0)}\right) \stackrel{D}{\rightarrow} N\left(0, \Sigma^{-1} \Gamma \Sigma^{-1}\right) .
$$

\section{Conclusion}

Combining the results of the above theoretical proof and numerical simulations, we can get the following conclusions.It is proved by theoretical properties that adoptive bridge estimation can accurately screen non-zero parameters with probability tending to 1 under high-dimensional data.Numerical simulations tested the performance of the proposed methods in a finite sample and verified the significance of modal regression estimation and the variable selection methods.

\section{References}

[1] Hastie, T. J, Tibshirani, R. J. (2017). Generalized Additive Models. New York, Routledge. doi: 10.1201/9780203753781.

[2] Guo, J, Tang, M. L, Tian, M. Z, Zhu, K. (2013).Variable selection in high-dimensional partially linear additive models for composite quantile regression. Computational Statistics and Data Analysis, 65 (9), $56 . \quad$ doi: 10.1016/j.csda.2013.03.017.

[3] Wang, L, Liu, X, Liang, H, Raymond, J. C. (2011). Estimation and variable selection for semiparmetric additive partial linear models. Statistica Sinica, 21 (3), 1225. doi: $10.2307 / 23033585$.

[4] Xia, Y. F, Qu, Y. R, Sun, N. L. (2018). Variable selection for semiparametric varying coefficient partially linear model based on modal regression with missing data. Communications in Statistics Theory and Methods, 48 (20), 5121. doi: 10.1080/03610926.2018.1508712.

[5] Fan, J. Q, Huang, T. (2005). Profile likelihood inferences on semiparametric varying-coefficient partially linear models. Bernoulli, 11 (6), 1031. doi: 10.2307/25464778.

[6] Hoshino, T. (2014). Quantile regression estimation of partially linear additive models. Journal of Nonparametric Statistics, 26 (3), 509.

doi: 10.1080/10485252.2014.929675.

[7] Meinshausen, N, Bhlmann, P. (2006). Highdimensional graphs and variable selection with the lasso. The Annals of statistics, 34 (3), 1436. doi: 10.1214/009053606000000281.

[8] Zhang, C. H, Huang, J. (2008). The sparsity and bias of the lasso selection in high-dimensional linear regression. The Annals of Statistics, 36 (4), 1567. doi: 10.1214/07AOS520.

[9] Wang, M. Q, Song, L. X, Wang, X. G. (2010). Bridge estimation for generalized linear models with a diverging number of parameters. Statistics Probability Letters, 80 (21), 1584. doi: 10.1016/j.spl.2010.06.012.

[10] Li, K. P, Li, D. G, Liang, Z. W. (2017). Estimation of semi-varying coefficient models with nonstationary regressors. Econometric Reviews, 36 (1), 354. doi: 10.1080/07474938.2015.1114563.

[11] Lam, C, Fan, J. Q. (2008). Profile-kernel likelihood inference with diverging number of parameters. The Annals of Statistics, 36(5), 2232. doi: 10.1214/07AOS544. 
[12] Yao, W. X, Lindsay, B. G, Li, R. Z. (2012). Local modal regression. Journal of Nonparametric Statistics, 24 (3), 647. doi: 10.1080/10485252.2012.678848.

[13] Fan, J. Q, Li, R. Z. (2001). Variable selection via nonconcave penalized likelihood and its oracle properties. Journal of the American statistical Association, 96(456), 1348. doi: 10.1198/016214501753382273.
[14] Zhao, P. X, Xue, L. G. (2009). Variable selection for semiparametric varying coefficient partially linear models. Statistics Probability Letters, 79(20), 2148. doi: 10.1016/j.spl.2009.07.004.

[15] Schumaker, L. L. (2007).Splines Function: Basic Theory. Cambridge, Cambridge University Press. doi:10.1017/CBO9780511618994. 